\title{
Priorisierung von bioziden Wirkstoffen aufgrund der potenziellen Gefährdung schweizerischer Oberflächengewässer
}

\author{
Daniel Bürgi · Lars Knechtenhofer · Isabel Meier ·Walter Giger
}

Erhalten: 26. Mai 2008/Akzeptiert: 13. November 2008/Online veröffentlicht: 10. Januar 2009

(C) Springer-Verlag 2009

\begin{abstract}
Zusammenfassung Hintergrund, Ziel und Fragestellungen Biozide Wirkstoffe können als Mikroverunreinigungen in Gewässern auftreten. Die heute in der Schweiz eingesetzten Biozide sollten daher in Bezug auf deren Umweltrisiko beurteilt und priorisiert werden. Folgende Fragestellungen standen im Vordergrund: Welche bioziden Wirkstoffe werden für welche Zwecke und in welchen Mengen verwendet? Gibt es Einträge in das Abwasser und in die Oberflächengewässer? Was sind Umweltverhalten und ökotoxikologische Auswirkungen der Biozide? Wie kann eine Priorisierung der zahlreichen Wirkstoffe erfolgen? Welche Biozide sollten für weitergehende Abklärungen vorgeschlagen werden?
\end{abstract}

Methoden Für die zur Diskussion stehende Problematik wurden Herstellerfirmen, Vekaufsorganisationen, Anwender und Umweltexperten als zentrale Akteure identifiziert und befragt. Dabei wurden chemische Identitäten, Produktzusammensetzungen, Einsatzmengen, Produktarten sowie Art und Ort der Anwendungen ermittelt. Die entsprechenden Informationen wurden in einer Datenbank mit Angaben zu 1760 Produkten mit insgesamt 277 unterschiedlichen Wirkstoffen erfasst. Für die Prioritätensetzung wurde ein mehrstufiges Priorisierungsverfahren bezüglich der Gewässergefährdung durch Biozide entwickelt. Dabei wurden mögliche Umweltemissionen sowie Angaben über

D. Bürgi $(\bowtie) \cdot$ L. Knechtenhofer · I. Meier

FRIEDLIPARTNER AG,

Baumackerstrasse, 8050 Zürich, Schweiz

E-Mail: Daniel.Buergi@friedlipartner.ch

W. Giger

GRC, Giger Research Consulting,

Im oberen Boden 128, 8049 Zürich, Schweiz

E-Mail: wgiger@hispeed.ch
Umweltverhalten und ökotoxikologische Eigenschaften berücksichtigt.

Ergebnisse In der Europäischen Union wurden 372 Substanzen als biozide Wirkstoffe notifiziert. Für die Schweiz konnte der Einsatz von 277 Substanzen ermittelt werden, die in einer Gesamtmenge von etwa 7500 Tonnen pro Jahr verbraucht werden. Mehr als $95 \%$ des Gesamtverbrauchs bzw. der Gesamtemission basieren auf ca. 30 Wirkstoffen, von denen sieben Substanzen biologisch schnell abbaubar sind. Eine Expertengruppe wählte 22 Substanzen bzw. Substanzgruppen als Kandidaten für eine weitergehende Beurteilung aus. Es zeigte sich, dass zahlreiche dieser Substanzen stark ökotoxisch für aquatische Organismen sind und bei den heute eingesetzten Mengen eine Umweltgefährdung bewirken könnten.

Diskussion Obwohl in der Schweiz gegen 300 biozide Wirkstoffe zur Anwendung kommen, werden nur etwa 35 Substanzen in relevanten Mengen verbraucht und emittiert (mehr als 15t/a Verbrauch bzw. mehr als 5t/a Emission pro Substanz). Für 22 dieser Wirkstoffe besteht aufgrund der schlechten Abbaubarkeit potenziell ein Risiko für eine Umweltgefährdung in Oberflächengewässern. Diese Substanzen werden in der Schweiz insgesamt in vergleichbaren Mengen wie die Pestizide eingesetzt.

Empfehlungen und Ausblick Im Hinblick auf die - im Vergleich zu den primär landwirtschaftlich eingesetzten Pestiziden - sehr schlechte Datenlage sind für die Biozide nähere Abklärungen zu Einsatz, Umweltverhalten und Ökotoxizität der prioritären Substanzen anzustreben. Für die Anwendung der Biozide sollten Handlungsempfehlungen erarbeitet werden, die sich an der Best Practice für die spezifischen Produktarten orientieren.

Schlüsselwörter Biozide $\cdot$ Wirkstoffe $\cdot$ Produktarten · Priorisierung 


\section{Prioritization of biocidal active substances based on the assessment of environmental risks in natural waters in Switzerland}

Abstract Background, Aim and Scope Active substances for use in biocidal products can potentially occur as micropollutants in natural waters. Biocides, which are used in Switzerland for non-agricultural applications, should be evaluated and prioritized with regard to their respective environmental risks. The following questions were emphasized: Which active substances are used for which purposes and in which amounts? Is there discharge to the aquatic environment? What are the environmental behaviours and ecotoxicological effects of these biocides? How can a prioritization be achieved? Which active substances should be suggested for further assessments?

Methods Manufacturing firms, sales organisations, users and environmental experts were identified as key stakeholders for the issues of this project. Chemical identities, product compositions, used amounts, product types, ways and places of applications were elucidated. A database was established with information on approximately 1760 products with totally 277 active substances. A prioritization procedure was established, providing a distinction of the active substances. For priority setting, water pollution risks by biocides were evaluated based on potential environmental emissions as well as on environmental behaviour and ecotoxic effects.

Results In the European Union 372 active substances were notified for biocidal products, which accounted for use of 7500 tons per year in Switzerland. More than $95 \%$ of the total use bases on 30 active substances, of which seven are rapidly biodegradable. A group of experts determined 22 substances as candidates for an extended assessment. It became evident that many active biocidal substances are highly ecotoxic und could, therefore, cause an augmented environmental risk

Discussion It could be shown which active substances are applied in Switzerland in substantial amounts. Although close to 300 active biocidal substances are used, only about 30 substances are employed in relevant amounts of more than $5000 \mathrm{~kg}$ per year. For 22 active substances with low biodegradability, a potential environmental risk for natural surface waters can be inferred. These active substances are used at amounts comparable to the pesticides.

Recommendations and Perspectives Looking at the - in comparison to the pesticides - very scarce knowledge for biocidal products, it is necessary to have a closer look at usages, environmental behaviour and ecotoxicity of the priority substances. For the practical application of the biocides, consulting should be given which is orientated on a best-practice procedure for the particular products.

Keywords Biocides $\cdot$ Biocidal products $\cdot$ Prioritization

\section{Einleitung}

Die heutige Zivilisation benutzt eine grosse Zahl technisch synthetisierter Chemikalien für vielfältige Anwendungsbereiche. Ungefähr 100.000 verschiedene Substanzen werden heute hergestellt. Einige von ihnen können Mensch und Umwelt gefährden. Für gewisse Chemikaliengruppen wie beispielsweise Pflanzenschutzmittel oder Wasch- und Reinigungsmittel sind seit längerer Zeit gesetzliche Vorschriften in Kraft. Für Zusatzstoffe wie zum Beispiel Formulierungsmittel, Konservierungsmittel, Weichmacher, Duftstoffe, Flammschutzmittel oder Korrosionsschutzsubstanzen fehlen Umweltschutz-Vorschriften jedoch weitgehend.

Auch für die Biozide bestanden bis vor wenigen Jahren keine entsprechenden Regelungen. Am 16. Februar 1998 erliess das Europäische Parlament und der Rat die Richtlinie 98/8/EG über das „Inverkehrbringen von Biozid-Produkten" (Europäische Gemeinschaft 1998). Diese sogenannte EG-Biozidprodukte-Richtlinie (EG-BPRL) schreibt ein Zulassungsverfahren für nichtlandwirtschaftlich eingesetzte Biozidpodukte vor und ist seit dem 14. Mai 1998 in Kraft. In Deutschland erfolgte die Überführung in nationales Recht am 28. Juni 2002. Das deutsche Biozidgesetz integriert die grundsätzlichen Vorschriften der EG-BPRL-Richtlinie wie Zulassungsbedürftigkeit, Zulassungsverfahren und Zulassungsvoraussetzungen in das Chemikaliengesetz und fasst diese in einem eigenen Abschnitt zusammen. Seit dem 1. August 2005 sind auch in der Schweiz die Biozidprodukte geregelt, und zwar in der Schweizerischen „Verordnung über das Inverkehrbringen von und den Umgang mit Biozidprodukten“ (Biozidprodukteverordnung, CH-VBP; Schweizerischer Bundesrat, 2005). Die Schweizerische Verordnung übernimmt die Vorschriften und Massnahmen der EG-Biozidprodukte-Richtlinie.

Gemäss Definitionen in der EG-BPRL bzw. in der CH-VBP sind Biozidprodukte Wirkstoffe und Zubereitungen, die dazu dienen, auf chemischem oder biologischem Wege Schadorganismen (Bakterien, Pilze, Insekten, Nagetiere etc.) abzuschrecken, unschädlich zu machen oder zu zerstören. Mit Biozidprodukten sollen Schädigungen von Lebensmitteln, Gebrauchsgegenständen, Baumaterialien (z. B. Holz) und anderen Produkten verhindert werden. $\mathrm{Zu}$ den Biozidprodukten gehören beispielsweise Desinfektionsmittel aller Art, Konservierungsmittel, Schutzmittel für Lebens- und Futtermittel, Holzschutzmittel und Unterwasseranstriche. Die EG-BPRL und die CH-VBP definieren 23 Produktarten (PA 1-PA 23), die in 4 Hauptgruppen aufgeteilt sind (HA 1-HA 4; siehe Tabelle 1). Nicht als Biozidprodukte bezeichnet werden Anwendungen, welche bereits in anderen Regelwerken geregelt sind (z.B. Pestizide im landwirtschaftlichen Bereich, Arzneimittel etc., vgl. Abschn. 1.1). 
Tabelle 1 Die vier Hauptgruppen (HG 1-HG 4) und 23 Produktarten (PA 1-PA 23) gemäss den europäischen und Schweizerischen Vorschriften über Biozidprodukte

\section{HG 1 Desinfektionsmittel}

1 Biozidprodukte für die menschliche Hygiene

2 Desinfektionsmittel für den Privatbereich und den Bereich des öffentlichen Gesundheitswesens sowie andere Biozidprodukte

3 Biozidprodukte für die Hygiene im Veterinärbereich

4 Desinfektionsmittel für den Lebens- und Futtermittelbereich

5 Trinkwasserdesinfektionsmittel

\section{HG 2 Schutzmittel}

6 Topf-Konservierungsmittel

7 Beschichtungsschutzmittel

8 Holzschutzmittel

9 Schutzmittel für Fasern, Leder, Gummi und polymerisierte Materialien

10 Schutzmittel für Mauerwerk

11 Schutzmittel für Flüssigkeiten in Kühl- und Verfahrenssystemen

12 Schleimbekämpfungsmittel

13 Schutzmittel für Metallbearbeitungsflüssigkeiten

HG 3 Schädlingsbekämpfungsmittel

14 Rodentizide

15 Avizide (Vogelbekämpfung)

16 Molluskizide (Schneckenbekämpfung)

17 Fischbekämpfungsmittel

18 Insektizide, Akarizide und Produkte gegen andere Arthropoden

19 Repellentien und Lockmittel

HG 4 Sonstige Biozidprodukte

20 Schutzmittel für Lebens- und Futtermittel

21 Antifouling-Produkte

22 Flüssigkeiten für Einbalsamierung und Taxidermie

23 Produkte gegen sonstige Wirbeltiere

Bei vielen Biozid-Produktarten ist eine signifikante Emission in das aquatische System gegeben. Dies ist insbesondere der Fall für Biozide, die direkt im Wasser oder in Kontakt mit Wasser eingesetzt werden, wie beispielsweise Desinfektionsmittel für Schwimmbäder, Konservierungsstoffe für Detergentien (Wasch- und Reinigungsmittel, gewisse Körperpflegemittel wie Shampoos oder Badezusätze), Biozide für Kühlwassersysteme oder Antifouling-Produkte in Bootsanstrichen. Emissionen in die aquatische Umwelt treten aber auch indirekt auf, wie z. B. bei Desinfektionsmittel für Oberflächen im privaten und öffentlichen Bereich (via das Waschwasser) oder bei Konservierungsmitteln für Lacke und Farben (via Fassadenabfluss). Im Zusammenhang mit dem Erlass der EG-BPRL wurden für die verschiedenen Produktarten Emissionsszenarien entwickelt, mit deren Hilfe die Emission einer Substanz für bestimmte Anwendungen abgeschätzt werden kann (siehe beispielsweise van der Poel und Bakker 2001).

Aufgrund von Emissionen in aquatische Umweltsysteme können Biozide als Mikroverunreinigungen in Abwasser und
Gewässer auftreten. Die bezüglich ihrer Gewässergefährdung weitaus am besten untersuchten nicht-landwirtschaftlichen Biozide sind die hauptsächlich in Bootsanstrichen eingesetzten organischen Zinnverbindungen (Fent 2006). Im Vordergrund steht dabei der Einsatz der Substanz Tributylzinn (TBT) als Antifouling im marinen Bereich (AntizarLadislao 2008), aber auch Flüsse und Seen sind betroffen (Fent 2006). Organische Zinnverbindungen gelangen über andere Verwendungen auch in die kommunalen Abwasser und Klärschlämme (Plagellat et al. 2004). Einige weitere Biozide sind in Siedlungsabwasser, Klärschlämmen und Gewässern untersucht worden. Dazu gehören Irgarol, Diuron und Sea-Nine 211 (Konstantinou und Albanis 2004), Triclosan und Triclocarban (Singer et al. 2002, Lindström et al. 2002, Heidler et al. 2006), Permethrin und Carbendazim (Kupper et al. 2006) sowie quartäre Ammoniumverbindungen (QAV; Martinez-Carballo et al. 2007, Clara et al. 2007).

Einsatzbedingt sind Biozide biologisch aktive Substanzen, die eine potenzielle Gefährdung für Umwelt und Mensch darstellen. Die EG-BPRL und CH-VBP sieht daher eine Beurteilung der Umweltverträglichkeit dieser Substanzen vor. Diese Beurteilung basiert auf der isolierten Bewertung jeder Anwendungsart einer Substanz. Es wird keine Gesamtbetrachtung einer Substanz über alle Produktarten gemacht. Die für die einzelnen Produktarten eingesetzten Emissionsszenarien sind zudem oft unvollständig. Von EGBPRL und CH-VBP ist zudem auch keine vergleichende Betrachtung der eingesetzten Wirkstoffe vorgeshen. Deshalb wird die Umweltverträglichkeit der Biozide in vorliegender Studie zusätzlich in einem übergreifenden und vergleichenden Sinne beurteilt werden. Ergänzend zum Ansatz in den bestehenden Regelungen wird dabei von der Gesamtexposition einer Substanz aufgrund der Anwendung in allen Produktarten ausgegangen.

Dieser Artikel gibt eine kurze Übersicht über das durchgeführte Projekt und die erzielten Ergebnisse. Ein ausführlicher Bericht kann auf dem Internet eingesehen werden (Bürgi et al. 2007). In einem anschliessenden Partner-Projekt wurden die Stoffflüsse über den gesamten Lebensweg für einige der hier als relevant beurteilten Substanzen abgeschätzt (Buser und Morf, 2008; Morf et al. 2007).

\section{Ziel und Fragestellungen}

Ziel des in diesem Artikel beschriebenen Projektes war die Beurteilung und Priorisierung der in der Schweiz eingesetzten Biozidprodukte und bioziden Wirkstoffe bezüglich einer Gewässergefährdung in der Schweiz. Dabei wurden folgende Fragen bearbeitet:

- Welche Produktarten und Wirkstoffe werden für welche Zwecke und in welchen Mengen eingesetzt? 
- Wo, wie und in welchem Umfang treten Emissionen in Abwasser und Gewässer auf?

- Wie verhalten sich die Biozide in der aquatischen Umwelt?

- Welche ökotoxikologischen Eigenschaften haben die Biozide?

- Wie können Produktarten und Wirkstoffe priorisiert werden?

- Welche Substanzen bzw. Stoffgruppen sollen näher untersucht werden?

- Welche zukünftige Abklärungen können vorgeschlagen werden?

\subsection{Abgrenzung}

In der durchgeführten Studie wurden nur biozide Anwendungen gemäss Definition in der EG-BPRL bzw. der CH-VBP betrachtet. Bei der Interpretation der Verbrauchs- und Emissionsdaten ist daher zu berücksichtigen, dass bei Einbezug weiterer Anwendungsbereiche für den gleichen Wirkstoff insgesamt höhere Mengen resultieren würden. In der vorliegenden Studie wurden insbesondere folgende Anwendungen nicht berücksichtigt, welche in anderen Regelwerken geregelt sind: Einsatz als Pestizide im landwirtschaftlichen Bereich, Einsatz als Wirkstoff in Arzneistoffen und Medizinprodukten, Einsatz in Kosmetika. Die Abgrenzung zwischen den entsprechen Regelwerken und der EG-BPRL bzw. $\mathrm{CH}-\mathrm{VBP}$ ist nicht immer eindeutig. Zur Klärung wurden von der EG zahlreiche sogenannte „Borderline“-Dokumente erarbeitet (vgl. http://europa.eu.int/comm/environment/ biocides/index.htm).

Die publizierten Schnittstellenregelungen klären aber nicht alle Fragen. Einerseits bestehen zahlreiche Überschneidungen von Regelungen (vgl. oben), andererseits ist die Regelung im
Biozidbereich nicht vollständig. Unter keine der erwähnten Regelungen fällt beispielsweise die Anwendung von bioziden Wirkstoffen im Materialschutz, z. B. in Dachbahnen (z. B. Mecoprop), obwohl Einsatz und Auswirkungen vergleichbar sind mit Biozid-Anwendungen in Anstrichstoffen, Holzschutzmitteln oder Mauerwerk (PA 7,8 und 10). In der Schweiz werden in Dachbahnen pro Jahr ca. 30 Tonnen Mecroprop eingesetzt, welche somit von der EG-BPRL bzw. der CH-VBP nicht erfasst werden (von Arx 1999). Von den erwähnten Biozidrichtlinien ausgenommen sind zudem fertig behandelte Artikel bzw. Materialien, welche von ausserhalb der EG importiert werden. Entsprechende Anwendungen wurden in der vorliegenden Studie ebenfalls nicht berücksichtigt.

\section{Methode}

Die Priorisierung der bioziden Wirkstoffe erfolgte über ein mehrstufiges Vorgehen, basierend auf den ermittelten Angaben zur Verwendung in der Schweiz, Abschätzungen der Einsatzmengen und der Emissionen in Abwasser und Gewässer sowie einer Beurteilung der Ökorelevanz beruhend auf Umweltverhalten und Ökotoxizität (siehe Abb. 1).

\subsection{Interviews und Literaturrecherchen}

In einem ersten Schritt wurden die für den Bereich Biozide zuständigen Akteure ermittelt. Die erstellte Adressliste umfasste mehr als 200 Herstellungs- und Formulierungsfirmen (Biozid-Wirkstoffe und Biozid-Produkte), Handelsfirmen, Branchenverbände, Anwender und Behörden sowie Hochschul- und Forschungsinstitute. Mit den wichtigsten Akteuren wurden aufgrund eines Fragebogens ungefähr zehn Interviews vor Ort und 135 telephonische Interviews geführt.

\begin{abstract}
Abb. 1 Schematischer Überblick über das in der vorliegenden Studie durchgeführte Priorisierungsverfahren für biozide Wirkstoffe, mit Angabe der Anzahl der Wirksubstanzen in den verschiedenen Stufen. Zum Vergleich ist auch die Anzahl der im Rahmen der EG-BPRL identifizierten und notifizierten Wirkstoffe dargestellt
\end{abstract}




Der in enger Zusammenarbeit mit Fachleuten des Schweizer Wasserforschungsinstituts Eawag in Dübendorf entwickelte Fragebogen umfasste Fragen zu den eingesetzten Biozid-Produkten und Biozid-Wirkstoffen (eingesetzte Konzentrationen, Mengen, Anwendung nach Produktart, Anwendungsart und Anwendungsorte etc.), zu möglichen Emissionen, zu Daten über Umweltverhalten und Ökotoxizität und zu einer möglichen Prioritätensetzung betreffend der Gewässergefährdung durch Biozide.

Viele Firmen verwiesen auf die auf den jeweiligen Firmen-Internetseiten enthaltenen umfangreichen Angaben. Diese öffentlich zugänglichen Daten wurden unter anderem bezüglich Produktinformationen, technischer Datenblätter und Sicherheitsdatenblätter ausgewertet. Eine Literaturstudie führte zu einer Sammlung mit 344 Publikationen. Von kommerziell erhältlichen, kostenpflichtigen Marktstudien im Bereich Biozide wurden einige zentrale Daten eingekauft. Allerdings haben sich die darin erhaltenen Angaben als sehr stark zusammengefasst und relativ ungenau erwiesen. Gemäss Aussagen eines Industrievertreters liegt die Ungenauigkeit der entsprechenden Angaben etwa bei einer Zehnerpotenz.

Vom European Chemicals Bureau (ECB, http://ecb.jrc.it/ biocides) werden im Zusammenhang mit der EG-BPRL zahlreiche Dokumente (Environmental Emmission Scenarios etc.) zur Verfügung gestellt, jedoch keine Verbrauchsdaten. Die EG-weiten Verbrauchsdaten liegen gemäss Aussagen der Biozid-Verantwortlichen auch beim ECB nicht in sinnvoll zusammengefasster Form vor. Auf nationaler Ebene konnten die vom Schweizer Bundesamt für Umwelt (BAFU) und vom Bundesamt für Gesundheit (BAG) erstellten Listen mit den zugelassenen Desinfektionsmitteln, Holzschutzmitteln und Antifoulingprodukten ausgewertet werden.

\subsection{Produktedatenbank und Abschätzung der Verbrauchsmengen}

Aufgrund der Ergebnisse von Interviews, Literatur- und Internetrecherchen wurde eine Datenbank mit Informationen zu 1761 Biozid-Produkten (CAS-Nummer, Konzentration, Produktart, Zusatzinfo) erstellt. Anschliessend wurde für jeden Wirkstoff die Verbrauchsmenge abgeschätzt. Zu Verbrauchsmengen einzelner biozider Wirkstoffe in der Schweiz liegen praktisch keine Daten vor. Die Verbrauchsmengen einzelner Wirkstoffe wurden daher aus den Gesamtverbräuchen aller Biozidprodukte in einer Produktart berechnet:

[Verbrauchsmenge von Wirkstoff X in Produktart Y]

$=[$ Verbrauch aller Biozidprodukte in Produktart $\mathrm{Y}]$

$\times$ [Marktanteil der Biozidprodukte mit Wirkstoff $\mathrm{X}$ in Produktart Y]

$\times$ [durchschnittliche Konzentration von Wirkstoff $\mathrm{X}$ im Biozidprodukt in Produktart Y]
Die Gesamtverbrauchsmenge von Wirkstoff $\mathrm{X}$ in allen Produktenarten zusammen ergibt sich durch Aufsummierung der Verbrauchsmengen in allen 23 Produktarten:

[Gesamtverbrauch von Wirkstoff X]

[2] $=\Sigma_{\mathrm{n}=1 \text { bis } 23}[$ Verbrauchsmenge von Wirkstoff $\mathrm{X}$ in Produktart n]

Die Inputdaten zu obenstehenden Berechnungen wurden im Rahmen der vorliegenden Studie recherchiert. Zum Verbrauch aller Biozidprodukte in einer Produktart konnten via Interviews und Literatur verwertbare Daten ermittelt werden. Beim Fehlen entsprechender Daten wurden „Bottom-Up“-Berechnungen durchgeführt (z. B. wurde der Gesamtverbrauch von Antifouling-Mittel berechnet aus dem Verbrauch pro Schiffsanstrich, der Anzahl Anwendungen pro Jahr und der Anzahl Schiffe in der Schweiz).

Angaben über die Marktanteile der jeweiligen Wirkstoffe konnten via Literatur oder Interviews gewonnen werden. Für die Hauptgruppe 1 (Desinfektionsmittel) konnten beim Schweizer Bundesamt für Gesundheit und via deutsche Fachstellen entsprechende Daten ermittelt werden. Auch für die Hauptgruppe 2 (Schutzmittel) konnten via Experteninterviews und Literaturangaben teilweise Angaben zu Marktanteilen von Einzelwirkstoffen gewonnen werden. Praktisch keine Daten zu Marktanteilen waren für die Hauptgruppen 3 und 4 vorhanden. Wo keine Angaben zum Marktanteil vorlagen, wurde dieser über die Einsatzhäufigkeit der jeweiligen Substanz in den ermittelten Produkten der jeweiligen Produktart abgeschätzt.

Die Konzentrationen der Wirkstoffe in den Biozidprodukten wurde aufgrund von Herstellerangaben, Expertenaussagen und Literaturdaten ermittelt. Die Herstellerangaben enthalten meistens nicht genaue Prozentangaben, sondern einen weit gefassten Konzentrationsbereich. In diesem Fall wurde für die Berechnungen ein Durchschnittswert (Best-Guess) eingesetzt. Wo keine genaueren Angaben aus Literatur oder Expertenbefragungen vorlagen, ist die Konzentrationsangabe daher mit grossen Unsicherheiten (z.T. mehr als eine Grössenordnung) behaftet.

\subsection{Unsicherheiten}

Alle erhobenen Verbrauchsdaten weisen beträchtliche Unsicherheiten auf (Bürgi et al. 2007). Für die meisten Produktarten konnten jedoch vergleichsweise verlässliche Mengenabschätzungen gemacht werden. Die Unsicherheit dieser Daten ist deutlich kleiner als eine Zehnerpotenz. Für PA 3 (Veterinärbereich), PA 4 (Lebens- und Futtermittel), PA 8 (Holzschutz) und PA 11 (Kühlsysteme) konnten hingegen nur sehr wenige Verbrauchsdaten ermittelt werden, bzw. die verwendeten Daten weisen eine grosse Unsicherheit auf. Die resultierenden Abschätzungen beinhalten daher eine Unsicherheit, die grösser als eine Zehnerpotenz sein dürfte. 
Tabelle 2 Dreistufiges Priorisierungsverfahren

\begin{tabular}{|c|c|c|c|c|}
\hline & & Hohe Relevanz & Mittlere Relevanz & Geringe Relevanz \\
\hline \multirow[t]{3}{*}{$\begin{array}{l}\text { Stufe 1: } \\
\text { Emission }\end{array}$} & $\begin{array}{l}\text { Emissionsmenge } \\
\text { in Abwasser (CH) }\end{array}$ & $>50 \mathrm{t} / \mathrm{a}$ & $>5 \mathrm{t} / \mathrm{a}$ & $<5 \mathrm{t} / \mathrm{a}$ \\
\hline & $\begin{array}{l}\text { Konzentration } \\
\text { in Gewässern, } \\
\text { ohne Abbau }\end{array}$ & $>1 \mu \mathrm{g} / \mathrm{L}$ & $>0.1 \mu \mathrm{g} / \mathrm{L}$ & $<0.1 \mu \mathrm{g} / \mathrm{L}$ \\
\hline & $\begin{array}{l}\text { Unsicherheit der } \\
\text { Mengenangabe }\end{array}$ & $\begin{array}{l}\text { Mengen evtl. unter- } \\
\text { schätzt }\end{array}$ & $\begin{array}{l}\text { Mengen wahrschein- } \\
\text { lich richtig geschätzt }\end{array}$ & $\begin{array}{l}\text { Mengen evtl. über- } \\
\text { schätzt }\end{array}$ \\
\hline $\begin{array}{l}\text { Stufe 2: } \\
\text { Umwelt- } \\
\text { verhalten/ } \\
\text { Exposition }\end{array}$ & Abbau/Persistenz & $\begin{array}{l}\text { kein bzw. sehr lang- } \\
\text { samer Abbau } \\
\text { (not biodegradable, } \\
\mathrm{t}_{1 / 2} \text { Wasser }>50 \\
\text { Tage) }\end{array}$ & $\begin{array}{l}\text { langsamer Abbau } \\
\text { (inherently bio- } \\
\text { degradable, } \mathrm{t}_{1 / 2} \\
\text { Wasser }>5 \text { Tage) }\end{array}$ & $\begin{array}{l}\text { schneller Abbau } \\
\text { (readily bio- } \\
\text { degradable, } \mathrm{t}_{1 / 2} \\
\text { Wasser }<5 \text { Tage) }\end{array}$ \\
\hline \multirow[t]{2}{*}{$\begin{array}{l}\text { Stufe 3: } \\
\text { Ökotoxizität }\end{array}$} & Bioakkumulation & $\begin{array}{l}\log \mathrm{K}_{\text {ow }}>4 \\
\mathrm{BCF}>500\end{array}$ & $\begin{array}{l}\log \mathrm{K}_{\mathrm{ow}}>2 \\
\mathrm{BCF}>100\end{array}$ & $\begin{array}{l}\log \mathrm{K}_{\mathrm{ow}}<2 \\
\mathrm{BCF}<100\end{array}$ \\
\hline & Aquatische Toxizität & $\begin{array}{l}\mathrm{EC}_{50} / \mathrm{LC}_{50} \\
<1 \mathrm{mg} / \mathrm{L} \\
\mathrm{NOEC}<0.1 \mathrm{mg} / \mathrm{L}\end{array}$ & $\begin{array}{l}\mathrm{EC}_{50} / \mathrm{LC} C_{50} \\
<10 \mathrm{mg} / \mathrm{L} \\
\mathrm{NOEC}<1 \mathrm{mg} / \mathrm{L}\end{array}$ & $\begin{array}{l}\mathrm{EC}_{50} / \mathrm{LC} C_{50} \\
>10 \mathrm{mg} / \mathrm{L} \\
\mathrm{NOEC}>1 \mathrm{mg} / \mathrm{L}\end{array}$ \\
\hline
\end{tabular}

Diese Unsicherheiten müssen bei der Diskussion und Verwertung der Daten berücksichtigt werden.

\subsection{Priorisierung}

Für die anschliessende Priorisierung der 277 in der Schweiz eingesetzten Wirkstoffe wurde ein dreistufiges Priorisierungsverfahren benutzt, das auf den Kriterien Emissionsmengen, Umweltverhalten und Ökotoxizität basiert (siehe Tabelle 2).

Im ersten Priorisierungsschritt wurden aus der Gesamtmenge der 277 in der Schweiz eingesetzten Wirkstoffe diejenigen Wirkstoffe ausgewählt, welche gemäss den Emissionsabschätzungen der vorliegenden Studie in erheblichen Mengen ( $>5 \mathrm{t} / \mathrm{a}$ ) emittiert werden.

Für die in hohen Mengen emittierten Substanzen wurden in einem zweiten Priorisierungsschritt das Umweltverhalten, insbesondere das Abbauverhalten beurteilt. Gut abbaubare Substanzen (Ethanol, Chlor etc.) wurden im weiteren Projektverlauf nicht näher betrachtet. Aufgrund der Emissionsmenge und des Abbauverhaltens wurden in einer ersten Expertenrunde potenziell umweltgefährdende Wirkstoffe zur näheren Betrachtung ausgewählt.

Für die ausgewählten, sogenannten Kandidaten-Substanzen, wurden chemisch-physikalische und ökotoxikologische Daten ermittelt. In einer zweiten Expertendiskussion wurden die vorhandenen Daten zu den Kandidaten-Wirkstoffen erörtert. Aufgrund der abgeschätzten Umweltgefährdung und zusätzlicher Kritierien (Metaboliten, Expositionsmessungen, Datenlage, Einsatz in anderen Anwendungen ausserhalb des Biozidbereichs, etc.) wurden schliesslich im dritten Priorisierungsschritt drei Prioritätsgruppen für die nähere Betrachtung der Kandidaten-Substanzen gebildet.

\section{Ergebnisse und Diskussion}

\subsection{Wirkstoffe und Verbrauchsmengen}

In der EG wurden 372 Substanzen als biozide Wirkstoffe notifiziert. Für die Schweiz konnte der Einsatz von 277 Substanzen ermittelt werden. Insgesamt werden diese bioziden Wirkstoffe in der Schweiz in einer Menge von ungefähr 7500 Tonnen pro Jahr eingesetzt. In Tabelle 3 werden die ermittelten Verbrauchsmengen pro Produktart und pro Hauptgruppe zusammengefasst. Ungefähr $75 \%$ der Verbrauchsmenge werden in der Schweiz in Hauptgruppe 1 (Desinfektionsmittel) eingesetzt, etwa $25 \%$ in Hauptgruppe 2 (Schutzmittel). In Hauptgruppe 3 (Schädlingsbekämpfungsmittel) und Hauptgruppe 4 (sonstige Biozidprodukte) werden nur kleine Mengen verbraucht (deutlich weniger als $15 \mathrm{t} / \mathrm{a}$ ).

$\mathrm{Zu}$ Vergleichszwecken enthält Tabelle 3 auch die Verbrauchsdaten für Dänemark (Lassen et al. 2001). Die zitierte dänischen Studie ist die einzige publizierte Arbeit, in der ebenfalls Verbrauchsmengen von Bioziden pro Produktart ermittelt wurden.

In Tabelle 4 sind die Verbrauchsmengen der 30 in der Schweiz am häufigsten verwendeten Wirkstoffe aufgeführt. Diese 30 Wirkstoffe bzw. Wirkstoffgruppen verursachen mehr als $95 \%$ der gesamten Biozid-Verbrauchsmenge in der Schweiz. Die restlichen ca. 250 ermittelten Substanzen werden nur in marginalen Mengen eingesetzt.

Von den in Tabelle 4 aufgeführten Substanzen sind die Alkohole (Ethanol, Propanol) und die schnell abbaubaren oxidativen Biozide (Chlor, Ozon, Peroxide) alleine bereits für ca. 2/3 des Verbrauchs verantwortlich. Der Verbrauch der restlichen, schlecht abbaubaren Wirkstoffe liegt mit 2000 bis $3000 \mathrm{t}$ aber immer noch in der gleichen Grössen- 
Tabelle 3 Verbrauchsmengen biozider Wirkstoffe in der Schweiz und in Dänemark

\begin{tabular}{|c|c|c|c|c|c|c|}
\hline \multirow[t]{2}{*}{$\begin{array}{l}\text { Haupt- } \\
\text { gruppe }\end{array}$} & \multicolumn{2}{|c|}{$\begin{array}{l}\text { Produktart gemäss } \\
\text { Biozidprodukteverordnung }\end{array}$} & \multicolumn{2}{|c|}{ Verbrauch $\mathbf{C H}^{\text {a) }}$} & \multicolumn{2}{|c|}{ Verbrauch DK ${ }^{\text {b) }}$} \\
\hline & & & {$[\mathbf{t} / \mathbf{a}]$} & {$[\%]$} & {$[\mathbf{t} / \mathbf{a}]$} & {$[\%]$} \\
\hline \multirow{5}{*}{ 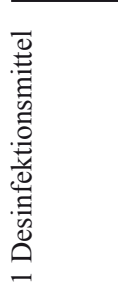 } & 1 & Menschliche Hygiene & 600 & 8 & 78 & 2 \\
\hline & 2 & $\begin{array}{l}\text { Desinfektionsmittel Privatbereich und Bereich des } \\
\text { öffentlichen Gesundheitswesens sowie andere Biozid- } \\
\text { produkte }\end{array}$ & 3800 & 51 & 2338 & 51 \\
\hline & 3 & Hygiene im Veterinärbereich & 1000 & 14 & 91 & 2 \\
\hline & 4 & $\begin{array}{l}\text { Desinfektionsmittel für den Lebens- und Futtermittel- } \\
\text { bereich }\end{array}$ & 150 & 2 & 593 & 13 \\
\hline & 5 & Trinkwasserdesinfektionsmittel & 1 & $<0.1$ & 41 & 0.9 \\
\hline \multicolumn{3}{|c|}{ Summe Hauptgruppe 1} & 5550 & 75 & 3141 & 69 \\
\hline \multirow{8}{*}{  } & 6 & Topf-Konservierungsmittel & 20 & 0.3 & 231 & 5 \\
\hline & 7 & Beschichtungsschutzmittel & 140 & 2 & 95 & 2 \\
\hline & 8 & Holzschutzmittel & 1100 & 15 & 434 & 10 \\
\hline & 9 & $\begin{array}{l}\text { Schutzmittel für Fasern, Leder, Gummi und poly- } \\
\text { merisierte Materialien }\end{array}$ & 20 & 0.3 & 94 & 2 \\
\hline & 10 & Schutzmittel für Mauerwerk & 20 & 0.3 & 18 & 0.4 \\
\hline & 11 & $\begin{array}{l}\text { Schutzmittel für Flüssigkeiten in Kühl- und Verfahrens- } \\
\text { systemen }\end{array}$ & 375 & 5 & 14 & 0.3 \\
\hline & 12 & Schleimbekämpfungsmittel & 100 & 1.5 & 123 & 3 \\
\hline & 13 & Schutzmittel Metallbearbeitungsflüssigkeiten & 50 & 0.7 & 14 & 0.3 \\
\hline \multicolumn{3}{|c|}{ Summe Hauptgruppe 2} & 1800 & 25 & 1023 & 22 \\
\hline \multirow{6}{*}{ 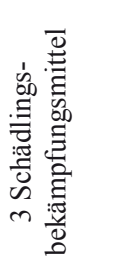 } & 14 & Rodentizide & $<0.1$ & $<0.01$ & 4.1 & 0.1 \\
\hline & 15 & Avizide (Vogelbekämpfung) & 0 & 0 & 0 & 0 \\
\hline & 16 & Molluskizide (Schneckenbekämpfung) & 0.7 & 0.01 & 0 & 0 \\
\hline & 17 & Fischbekämpfungsmittel & 0 & 0 & 0 & 0 \\
\hline & 18 & $\begin{array}{l}\text { Insektizide, Akarizide und Produkte gegen andere } \\
\text { Arthropoden }\end{array}$ & 0.3 & $<0.01$ & 9.1 & 0.2 \\
\hline & 19 & Repellentien und Lockmittel & 1.6 & 0.02 & 3.7 & 0.1 \\
\hline \multicolumn{3}{|c|}{ Summe Hauptgruppe 3} & 3 & 0.05 & 17 & 0.4 \\
\hline \multirow{4}{*}{ 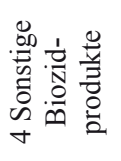 } & 20 & Schutzmittel Lebens- und Futtermittel & 0 & 0 & 0 & 0 \\
\hline & 21 & Antifouling-Produkte & 13 & 0.2 & 360.6 & 8 \\
\hline & 22 & Flüssigkeiten zur Einbalsamierung/Taxidermie & 0.2 & $<0.01$ & 13.7 & 0.3 \\
\hline & 23 & Produkte gegen sonstige Wirbeltiere & 0 & 0 & 4.1 & 0.1 \\
\hline \multicolumn{3}{|c|}{ Summe Hauptgruppe 4} & 13 & 0.2 & 378 & 8 \\
\hline \multicolumn{3}{|c|}{ Summe Total } & 7400 & 100 & 4565 & 100 \\
\hline
\end{tabular}

Alle Daten enthalten signifikante Unsicherheiten (vgl. Abschnitt 2.3).

a) Diese Studie b) Lassen et al., 2001

ordnung wie der Verbrauch an Pestiziden in der Schweiz (ca. 1400t organische Wirkstoffe für Pflanzenschutzmittel im Jahre 2004; SGCI 2005).

\subsection{Priorisierung}

Priorisierungsstufe 1 aufgrund der Emissionsmenge. Aus den Verbrauchsdaten wurde mittels eines (für jede Produktart separat abgeschätzten) Emissionsfaktors die Emissionsmengen in die aquatische Umwelt berechnet. Bei der Auswertung der Emissionsmengen zeigte sich, dass nur 35 Substanzen eine Emission von je mehr als 5 t/a aufweisen.
Diese Substanzen machen zusammen bereits mehr als 95\% der Gesamtemission aus und wurden in einem ersten Priorisierungsschritt zur näheren Betrachtung ausgewählt.

Priorisierungsstufe 2 aufgrund der Abbaubarkeit. Für die 35 bioziden Wirkstoffen, welche in relevanten Mengen emittiert werden ( $>5 \mathrm{t} / \mathrm{a}$ ), wurden Daten zur Abbaubarkeit ermittelt. Wirkstoffe, welche schnell abbaubar sind (Ozon, Peroxide, Chlor, Ethanol, etc.) wurden im weiteren Projektverlauf nicht näher betrachtet. Die restlichen Wirkstoffe sind potenziell umweltrelevant, da sie in relevanten Mengen eingesetzt und freigesetzt werden, sowie nicht gut abbaubar sind. 
Tabelle 4 Verbrauchsmengen der 30 in der Schweiz am häufigsten verwendeten bioziden Wirkstoffe bzw. Wirkstoffgruppen

\begin{tabular}{|c|c|c|c|c|c|c|}
\hline \multirow[t]{2}{*}{ Wirkstoff } & \multirow{2}{*}{$\begin{array}{c}\text { Verbrauch } \\
{[\mathbf{t} / \mathbf{a}]}\end{array}$} & \multirow{2}{*}{$\begin{array}{c}\text { Anteil am } \\
\text { Gesamt- } \\
\text { verbrauch } \\
{[\%]}\end{array}$} & \multicolumn{4}{|c|}{ Verbrauch pro Hauptgruppe [t/a] } \\
\hline & & & HG 1 & HG 2 & HG 3 & HG 4 \\
\hline Ethanol/Propanol & 3400 & 46 & 3383 & 0 & 0 & 0 \\
\hline Chlor & 900 & 12 & 682 & 220 & 0 & 0 \\
\hline Borsäure und Borate & 590 & 8 & 0 & 590 & 0 & 0 \\
\hline Glutaraldehyd & 390 & 5 & 372 & 21 & 0 & 0 \\
\hline Organische Säuren & 290 & 4 & 286 & 2 & 0 & 0 \\
\hline $\begin{array}{l}\text { Quartäre Ammonium- } \\
\text { verbindungen (QAV) }\end{array}$ & 210 & 3 & 127 & 83 & 0 & 0 \\
\hline Ozon & 210 & 3 & 133 & 73 & 0 & 0 \\
\hline Hypochlorit & 170 & 2 & 105 & 66 & 0 & 0 \\
\hline Guanidin-Derivate & 170 & 2 & 163 & 6 & 0 & 0 \\
\hline Octylisothiazolinon (OIT) & 100 & 1 & 0 & 104 & 0 & 0 \\
\hline Dichlofluanid & 100 & 1 & 0 & 103 & 0 & 0 \\
\hline $\mathrm{CMI} / \mathrm{MI}^{\mathrm{a})}$ & 90 & 1 & 37 & 54 & 0 & 0 \\
\hline $\mathrm{IPBC}^{\mathrm{b})}$ & 75 & 1 & 0 & 76 & 0 & 0 \\
\hline Formaldehyd & 75 & 1 & 74 & 0 & 0 & 0 \\
\hline Zinkoktoat & 70 & 0.9 & 0 & 69 & 0 & 0 \\
\hline Peroxid-Verbindungen & 50 & 0.7 & 48 & 1 & 0 & 0 \\
\hline Aldehyde (diverse) & 45 & 0.6 & 45 & 0 & 0 & 0 \\
\hline Alkylamine & 45 & 0.6 & 44 & 0 & 0 & 0 \\
\hline Tributylzinn-Naphtenat & 40 & 0.5 & 0 & 39 & 0 & 0 \\
\hline Propiconazol & 35 & 0.5 & 0 & 36 & 0 & 0 \\
\hline Chlordioxid & 35 & 0.5 & 34 & 0 & 0 & 0 \\
\hline Tolylfluanid & 35 & 0.5 & 0 & 33 & 0 & 0 \\
\hline Tebuconazol & 30 & 0.4 & 0 & 31 & 0 & 0 \\
\hline Terbutryn & 30 & 0.4 & 0 & 27 & 0 & 0 \\
\hline Carbendazim & 25 & 0.3 & 0 & 26 & 0 & 0 \\
\hline Irgarol & 25 & 0.3 & 0 & 24 & 0 & 0 \\
\hline Diuron & 25 & 0.3 & 0 & 24 & 0 & 0 \\
\hline Benzisothiazolinon (BIT) & 20 & 0.3 & 0 & 22 & 0 & 0 \\
\hline Bronopol & 20 & 0.3 & 4 & 16 & 0 & 0 \\
\hline Zink-Pyrithion & 20 & 0.3 & 0 & 19 & 0 & 0 \\
\hline Total & 7300 & 100 & 5500 & 1700 & $<1$ & $<1$ \\
\hline
\end{tabular}

Alle Daten enthalten signifikante Unsicherheiten (vgl. Abschnitt 2.3).

a) $\mathrm{CMI}=5$-Chloro-2-methylisothiazolin-3-on. $\mathrm{MI}=2$-Methylisothiazolin-3-on

b) IPBC = 3-Iodoprop-2-ynyl-N-butylcarbamat
Aufgrund der Angaben zu Emissionsmengen und Abbau sowie aufgrund einer ersten Einschätzung durch eine Expertengruppe, wurden schliesslich die in Tabelle 5 aufgeführten 22 Substanzen bzw. Substanzgruppen als Kandidaten für eine nähere Betrachtung ausgewählt. Bei chemisch verwandten Substanzen wurde nur ein Vertreter näher betrachtet, z. B. BAC C12-C18 stellvertretend für die quartären Ammoniumverbindungen oder Dichlofluanid stellvertretend für die Fluanide, siehe Tabelle 5.

Priorisierungsstufe 3 aufgrund von Umweltverhalten und Ökotoxizität. Für die in Tabelle 5 aufgeführten 22 Kandidatensubstanzen wurden chemisch-physikalische sowie ökotoxikologische Daten zusammengestellt und das Umweltrisiko abgeschätzt. Die Bewertung der Daten erfolg- te anhand des in Abschnitt 2.4 vorgestellten Priorisierungssystems (siehe Tabelle 2). Dabei zeigte sich, dass zahlreiche dieser Substanzen relativ stark toxisch für aquatische Organismen sind und die aquatische Umwelt gefährden könnten. In Tabelle 6 wird am Beispiel von vier Wirkstoffen die Bewertung der Daten zu Verbrauchsmengen, Exposition und Ökotoxizität dargestellt.

In einer Expertenrunde mit Vertretern des Schweizer Bundesamtes für Umwelt, von Entsorgung + Recycling Zürich (ERZ) sowie mit Vertretern der Arbeitsgemeinschaft GEOPartner, FRIEDLIPARTNER und Eawag wurden die oben erwähnten Daten diskutiert. Aufgrund dieser Angaben und zusätzlicher Kritierien (Metaboliten, Expositionmessungen, Umfang der vorhandenen Informationen, Einsatz in ande- 
Tabelle 5 Auswahl der 22 Kandidaten-Substanzen, welche aufgrund der hohen Verbrauchs- bzw. Emissionsmengen und des Umweltverhaltens (nicht gut abbaubar) von einer Expertengruppe als potenziell gefährdend für die Oberflächengewässer in der Schweiz eingestuft wurden

\begin{tabular}{|c|c|c|c|c|c|}
\hline Substanzname & Bemerkungen zur Auswahl der Substanz & EC-Nr. & CAS-Nr. & $\begin{array}{l}\text { Verbrauch } \\
\text { (t/a) }\end{array}$ & $\begin{array}{c}\text { Emission } \\
(\mathbf{t} / \mathbf{a})\end{array}$ \\
\hline Borsäure & Vertreter für Borsäure und Borate & $233-139-2$ & $10043-35-3$ & 590 & 295 \\
\hline Glutaraldehyd & & $203-856-5$ & $111-30-8$ & 393 & 197 \\
\hline $\mathrm{BAC}$ C12-C18 ${ }^{\text {a) }}$ & Vertreter der quartären Ammonium-Verbindungen (QAV) & 269-919-4 & $68391-01-5$ & 210 & 139 \\
\hline PHMB $^{\text {b) }}$ & Vertreter der Guanidin-Derivate & Polymer & $32289-58-0$ & 169 & 85 \\
\hline Octylisothiazolinon & Erster Vertreter der Isothiazolinone & $247-761-7$ & $26530-20-1$ & 104 & 53 \\
\hline Dichlofluanid & Vertreter der Fluanide (Dichlofluanid, Tolylfluanid & 214-118-7 & $1085-98-9$ & 103 & 52 \\
\hline Diethylamin & Vertreter der Alkylamine & & $109-89-7$ & 44 & 44 \\
\hline $\mathrm{IPBC}^{\mathrm{c})}$ & & $259-627-5$ & $55406-53-6$ & 76 & 38 \\
\hline Formaldehyd & & $200-001-8$ & $50-00-0$ & 74 & 37 \\
\hline Propiconazol & Vertreter der Triazole (Propiconazol, Tebuconazol) & $262-104-4$ & $60207-90-1$ & 36 & 18 \\
\hline Terbutryn & & $212-950-5$ & $886-50-0$ & 27 & 14 \\
\hline Carbendazim & & $234-232-0$ & $10605-21-7$ & 26 & 13 \\
\hline Irgarol & & $248-872-3$ & 28159-98-0 & 24 & 12 \\
\hline Diuron & & $206-354-4$ & $330-54-1$ & 24 & 12 \\
\hline Benzisothiazolinon & Zweiter Vertreter der Isothiazolinone & $220-120-9$ & $2634-33-5$ & 22 & 11 \\
\hline Bronopol & & $200-143-0$ & $52-51-7$ & 19 & 10 \\
\hline Zink-Pyrithion & Vertreter der Zink-Verbindungen & $236-671-3$ & $13463-41-7$ & 19 & 9 \\
\hline Permethrin & & 258-067-9 & $52645-53-1$ & 14 & 7 \\
\hline Kupferoxid & & $215-269-1$ & $1317-38-0$ & 6 & 6 \\
\hline DMDM Hydantoin & Vertreter der Imidazolidine & $229-222-8$ & $6440-58-0$ & 7 & 4 \\
\hline OBPA & Kandidat, da stark toxisch (arsenhaltig) & $200-377-3$ & $58-36-6$ & 1 & 0 \\
\hline Diethyltoluamid (DEET) & Kandidat aufgrund gemessener Immissionen & $205-149-7$ & $134-62-3$ & 1 & 0 \\
\hline Total & & & & 2000 & 1050 \\
\hline
\end{tabular}

a) BAC-C12: N-Dodecyl-N-benzyl-N,N-dimethylammoniumchlorid

b) $\mathrm{PHMB}=$ Polyhexamethylenbiguanidin

c) IPBC = 3-Iodoprop-2-ynyl-N-butylcarbamat

ren Anwendung ausserhalb des Biozidbereichs, etc.) wurden schliesslich drei Wirkstoffgruppen bzw. Wirkstoffe zur näheren Betrachtung in einer Stoffflussanalyse empfohlen. Dabei handelt es sich um die quartären Ammoniumverbindungen (QAV), Isothiazolinon-Verbindungen und Irgarol. Die endgültige Auswahl fiel in Absprache mit dem Auftraggeber auf die Gruppe der QAV. (Buser und Morf, 2008)

\section{Schlussfolgerungen}

Gemäss den Abschätzungen in der vorliegenden Studie werden in der Schweiz jährlich ca. $7500 \mathrm{t}$ biozide Wirkstoffe eingesetzt. Diese Menge liegt um ungefähr Faktor fünf höher als die entsprechenden Verbrauchsmenge der Pestizide. In Anbetracht dieser Mengenverhältnisse ist der Wissensstand über Einsatz und Umweltverhalten von Bioziden im Vergleich zu den Pestziden sehr gering. Hier besteht unseres Erachtens ein deutlicher Handlungsbedarf für die Umweltschutzbehörden. Diese Situation wird durch die EG-BPRL und die CH-VBP nur teilweise verbessert, weil damit zwar einzelne Wirkstoffe und Produkte umschrieben und geregelt werden, hingegen erfolgt keine vergleichende Betrachtung und keine Gesamtbetrachtungen der Exposition, die von den aquatischen Ökosystemen ausgeht.

Für die Schweiz wurde der Einsatz von 277 unterschiedlichen bioziden Wirkstoffen nachgewiesen. Eine detaillierte Untersuchung all dieser Substanzen wäre kaum zu bewältigen. In der vorliegenden Studie konnte der Umfang an näher zu betrachtenden Wirkstoffen auf 22 Substanzen eingegrenzt werden, die sowohl bezüglich der Verbrauchsbzw. Emissionsmengen als auch betreffend ihres Umweltverhaltens (schlechte Abbaubarkeit) als relevant einzustufen sind.

Aufgrund der Daten zu Umweltverhalten und Ökotoxizität der 22 Substanzen stellen einige dieser Substanzen potenziell eine Gefährdung der aquatischen Umwelt dar. Vermehrte Abklärungen in den Bereichen Anwendung und Umweltverhalten von Bioziden scheinen aufgrund der oben erwähnten Ausgangslage angebracht. Die vorliegende Studie bietet mehrere Ansatzpunkte, um zukünftig eine umweltverträgliche Biozidanwendung sicherstellen zu können (vgl. Abschnitt 5). 
Tabelle 6 Bewertung der Daten zu Verbrauchsmenge, Exposition und Ökotoxizität für die quartären Ammoniumverbindungen (QAV), Irgarol sowie für zwei Vertreter der Isothiazolinone. Die zutreffende Bewertung ist jeweils schattiert und fett hervorgehoben. Falls bei einer Eigenschaft zwei Bewertungen angegeben werden (z. B. bei der Exposition der QAV), so liegen unterschiedliche bzw. nicht eindeutige Daten vor

\begin{tabular}{|c|c|c|c|c|c|}
\hline \multirow[t]{2}{*}{ Substanz } & \multicolumn{2}{|c|}{ Mengen } & \multicolumn{2}{|c|}{ Exposition } & \multirow{2}{*}{$\begin{array}{l}\text { Ökotoxizität } \\
\mathrm{EC}_{50} / \mathrm{LC}_{50} \text { oder } \\
\text { NOEC oder } \\
\text { CQK oder PNEC }\end{array}$} \\
\hline & $\begin{array}{l}\text { Verbrauch, } \\
\text { Emission, } \\
\text { Theoretische } \\
\text { Konzentration }\end{array}$ & Unsicherheit & $\begin{array}{l}\text { Persistenz } \\
\text { (OSPAR- } \\
\text { Kriterien) }\end{array}$ & $\begin{array}{l}\mathrm{K}_{\mathrm{ow}} / \mathrm{BCF} \\
\text { (OSPAR- } \\
\text { Kriterien) }\end{array}$ & \\
\hline \multirow[t]{3}{*}{ QAV } & $>1 \mathrm{ug} / \mathrm{L}$ & $\begin{array}{l}\text { evt. unter- } \\
\text { schätzt }\end{array}$ & $>50 \mathrm{~d}$ & $\begin{array}{c}>4 / \\
>500\end{array}$ & hohe Tox. \\
\hline & $0.1-1 \mathrm{ug} / \mathrm{L}$ & wahrsch. richtig & 5-50 d & $\begin{array}{c}2-4 / \\
100-500\end{array}$ & mittlere Tox. \\
\hline & $<0.1 \mathrm{ug} / \mathrm{L}$ & evt. überschätzt & $<5$ d & $\begin{array}{l}<2 / \\
<100\end{array}$ & tiefe Tox. \\
\hline \multirow[t]{3}{*}{ Irgarol } & $>1 \mathrm{ug} / \mathrm{L}$ & evt. unterschätzt & $>50 \mathrm{~d}$ & $\begin{array}{c}>4 / \\
>500\end{array}$ & hohe Tox. \\
\hline & $0.1-1 \mathrm{ug} / \mathrm{L}$ & wahrsch. richtig & $5-50 d$ & $\begin{array}{c}2-4 / \\
100-500\end{array}$ & mittlere Tox. \\
\hline & $<0.1 \mathrm{ug} / \mathrm{L}$ & evt. überschätzt & $<5 \mathrm{~d}$ & $\begin{array}{l}<2 / \\
<100\end{array}$ & tiefe Tox. \\
\hline \multirow[t]{3}{*}{$\begin{array}{l}\text { Isothiazolinon } \\
\text { OIT }\end{array}$} & $>1 \mathrm{ug} / \mathrm{L}$ & evt. unterschätzt & $>50 \mathrm{~d}$ & $\begin{array}{c}>4 / \\
>500\end{array}$ & hohe Tox. \\
\hline & $0.1-1 \mathrm{ug} / \mathrm{L}$ & wahrsch. richtig & $5-50 \mathrm{~d}$ & $\begin{array}{c}2-4 / \\
100-500\end{array}$ & mittlere Tox. \\
\hline & $<0.1 \mathrm{ug} / \mathrm{L}$ & evt. überschätzt & $<5 \mathrm{~d}$ & $\begin{array}{l}<2 / \\
<100\end{array}$ & tiefe Tox. \\
\hline \multirow[t]{3}{*}{$\begin{array}{l}\text { Isothiazolinon } \\
\text { BIT }\end{array}$} & $>1 \mathrm{ug} / \mathrm{L}$ & evt. unterschätzt & $>50 \mathrm{~d}$ & $\begin{array}{c}>4 / \\
>500\end{array}$ & hohe Tox. \\
\hline & $0.1-1 \mathrm{ug} / \mathrm{L}$ & wahrsch. richtig & $5-50 \mathrm{~d}$ & $\begin{array}{c}2-4 / \\
100-500\end{array}$ & mittlere Tox. \\
\hline & $<0.1 \mathrm{ug} / \mathrm{L}$ & evt. überschätzt & $<5$ d & $\begin{array}{l}<2 / \\
<100\end{array}$ & tiefe Tox. \\
\hline
\end{tabular}

Legende:

Hohe Relevanz

Mittlere Relevanz

Geringe Relevanz

NOEC: No effect concentration

CQK: Chronische Qualitätskriterien

PNEC: Predicted no effect concentration

OIT: Octylisothiazolinon

BIT: Benzisothiazolinon

\section{Empfehlungen und Ausblick}

Aufgrund der potenziellen Umweltgefährdung sollten Einsatz und Umweltverhalten der 22 Kandidatensubstanzen (siehe Tabelle 5) näher untersucht werden. Aus Kapazitätsgründen konnten im Partner-Projekt mit den quartären Ammoniumverbindungen nur eine Substanzgruppe in einer Stoffflussanalyse für die Schweiz näher untersucht werden (Buser und Morf, 2008, Morf et al. 2007). In erster Priorität zur näheren Abklärung bezüglich Einsatz und Umweltverhalten werden im weiteren die Isothiazolinone und Irgarol empfohlen. Zur näheren Betrachtung in zweiter Priorität vorgeschlagen werden Bronopol, Dichlofluanid, Diuron, IPBC, PHMB, Propiconazol, Terbutryn und Zink-Pyrithion.
In eine dritte Priorität zur näheren Beurteilung eingeteilt werden Alkylamine, Borsäure, Carbendazim, Kupferoxid, DEET, Formaldehyd, Glutaraldehyd und Permethrin.

Die bioziden Wirkstoffe werden in den verschiedenen Produktarten auf sehr unterschiedliche Art eingesetzt. Neben der oben erwähnten substanzspezifischen Abklärung empfehlen wir daher, den Einsatz pro Produktart näher zu betrachten. Eine in einer Produktart gewünschte biozide Wirkung kann mit sehr unterschiedlichen Wirkstoffen erzielt werden. In einigen Fällen kann der gewünschte Schutz auf andere Weise erreicht und ganz auf biozide Wirkstoffe verzichtet werden. Zur Förderung eines professionellen und umweltschonenden Umgangs mit Bioziden empfehlen wir daher, die Best Practice pro Produktart zu ermitteln. Die 
entsprechenden Informationen sind anschliessend den Anwendern der jeweiligen Produktart zur Verfügung zu stellen. Die Erarbeitung der bewährten Praxis und die Zusammenstellung von Handlungsempfehlungen sollte in Zusammenarbeit mit den jeweiligen Branchenverbänden erfolgen.

Prioritär empfehlen wir insbesondere die nähere $\mathrm{Ab}$ klärung von Einsatz und Best Practice in den folgenden, mengenmässig bzw. bezüglich eingesetzter Substanzen relevanten Produktarten: Menschliche Hygiene (PA 1), Desinfektion privater und öffentlicher Bereich (PA 2), Veterinärhygiene (PA 3), Lebensmittel und Futtermittel (PA 4), Beschichtungsschutz (PA 7), Holzschutzmittel (PA 8), Kühlund Verfahrenssysteme (PA 11), Schleimbekämpfung (PA 12) und Antifouling (PA 21).

Falls sich bei der näheren Beurteilung einzelner Produktarten zeigt, dass der heutige Biozideinsatz nicht umweltgerecht erfolgt, sind - neben der Information über die Best Practice - weitere technische oder rechtliche Massnahmen zur Sicherstellung des umweltverträglichen Einsatzes in der entsprechenden Produktart zu prüfen. Um eine erste Sensibilisierung der betroffenen Branchen zu erreichen, empfehlen wir, die hier vorliegenden Ergebnisse mit Branchenvertretern - gegebenenfalls nach Produktart getrennt - zu diskutieren. Das weitere Vorgehen für einzelne Produktarten sollte in Absprache mit den Branchenvertretern festgelegt werden.

Danksagung Wir bedanken uns bei allen Kollegen, die uns durch das Ausfüllen der Fragebogen und durch Diskussionen geholfen haben, eine tragfähige Datenbank aufzubauen und das vorgeschlagene Priorisierungsverfahren durchzuführen. Finanziert wurde diese Studie durch das Schweizer Bundesamt für Umwelt (BAFU) im Rahmen des Projekts „MicroPoll - Erkennung und Priorisierung von Mikroverunreinigungen und mögliche Wandlung der heutigen Siedlungswasserwirtschaft" sowie durch die Abteilung Entsorgung + Recycling Zürich (ERZ) der Stadt Zürich.

\section{Literatur}

Antizar-Ladislao B (2008) Environmental levels, toxicity and human exposure to tributyltin (TBT)-contaminted marine environment. A review. Env Int 34:292-308

Bürgi D, Knechtenhofer L, Meier I (2007) Biozide als Mikroverunreinigungen in Abwasser und Gewässern - Teilprojekt 1: Priorisierung von Produktarten und Wirkstoffen, http://www.bafu. admin.ch/gewaesserschutz/03716/06387/index.html?lang=de (26.5.2008)

Buser A, Morf L (2008) Stoffflussanalyse von quartären Ammoniumverbindungen für die Schweiz. Modellierung des Verbrauchs in bioziden Anwendungen und der Emissionen in die Umwelt. Umweltwiss Schadst Forsch, DOI: 10.1007/s12302-008-0025-1
Europäische Gemeinschaft (1998) Richtlinie 98/8/EG des europäischen Parlaments und des Rates vom 16. Februar 1998 über das Inverkehrbringen von Biozid-Produkten. BPRL http://eur-lex.europa.eu/LexUriServ/site/de/oj/1998/1_123/ 1_12319980424de00010063.pdf

Fent $\overline{\mathrm{K}}$ (2006) Worldwide occurrence of organotins from antifouling paints. Hdb Env Chem $5(\mathrm{O}): 71-100$

Clara M, Scharf S, Scheffknecht C, Gans O (2007) Occurrence of selected surfactants in untreated and treated sewage. Water Res 41(19):4339-4348

Heidler J, Sapkota J, Halden RU (2006) Partitioning, persistence, and accumulation in digested sludge of the topical antiseptic triclocarban during wastewater treatment. Environ Sci Technol 40(11):3634-3639

Konstantinou IK, Albanis TA (2004) Worldwide ocurrence and effects of antifouling paint booster bioides in the aquatic environment: a review. Env Int 30(2):235-248

Kupper T, Plagellat C, Brändli RC, de Alencastro LF, Grandjean D, Tarradellas J (2006) Fate and removal of polycyclic musks, UV filters and biocides during wastewater treatment. Water Res 40(14):2603-2612

Lassen C. et al. (2001) Inventory of biocides used in Denmark. Environmental Project No 585 2001, Danish Environmental Protection Agency, Copenhagen

Lindström A, Buerge I., Poiger M, Mueller M, Buser HR (2002) Occurrence and environmental behavior of the bactericide triclosan and its methyl derivative in surface waters and in wastewater. Environ Sci Technol 36:2322-2329

Martinez-Carballo E, Sitka A, Gonzalez-Barreiro C, Kreuzinger N, Fürhacker M, Scharf, Gans O (2007) Determination of selected quaternary ammonium compounds by liquid chromatography with mass spectrometry. Part I. Application to surface, waste and indirect discharge water samples in Austria. Environ Poll 145:489-496

Morf L, Buser A, Gubler A (2007) Biozide als Mikroverunreinigungen in Abwasser und Gewässern - Teilprojekt 2: Stoffflussanalyse für die Schweiz: Quartäre Ammoniumverbindungen, http:/www.bafu.admin.ch/gewaesserschutz/03716/06387/index.html?lang=de (26.5.2008)

Plagellat C, Kupper T, de Alencastro LF, Grandjean D, Tarradellas J (2004) Biocides in Sewage Sludge: Quantitative Determination in Some Swiss Wastewater Treatment Plants. Bull Environ Contam Toxicol 73(5):794-801

Schweizerischer Bundesrat (2005): Verordnung über das Inverkehrbringen von und den Umgang mit Biozidprodukten (Biozidprodukteverordnung). http://www.admin.ch/ch/d/sr/8/813.12.de.pdf

SGCI (2005) SGCI Chemie Pharma Schweiz. Pflanzenschutzmittelstatistik 2004

Singer H, Mueller S, Tixier C, Pillonel L (2002) Triclosan: occurrence and fate of a widely used biocide in the aquatic environment: field measurements in wastewater treatment plants, surface waters, and lake sediments. Environ Sci Technol 36:4998-5004

van der Poel P, Bakker J (2001) Emission Scenario Document for Biocides: Emission scenarios for all 23 product types of the Biocidal Products Directive (EU Directive 98/8/EC). 601450009, RIVM. http://ecb.jrc.it/documents/Biocides/EMISSION_SCENARIO_DOCUMENTS/ADDITIONAL_INFORMATION/ESDs_ RIVM_2001_ESD_PT1-PT23.pdf

von Arx U (1999) Bauprodukte und -inhaltsstoffe, Bundesamt für Umwelt, Wald und Landschaft (BUWAL), Bern 